\title{
HISTORIA DE UNA PUBLICACIÓN PERIÓDICA: EL CASO DE LA REVISTA DRAGÓN (EDICIÓN NORTEAMERICANA)
}

\author{
History of a Journal: the Case of Dragon Magazine (U.S. Edition)
}

Héctor Sevillano Pareja

hsevillanopareja@gmail.com

Fecha de recepción: 15-III-2011

Fecha de aceptación: 6-V-2011

Resumen: El presente trabajo analizamos la historia y el desarrollo de una publicación periódica, desde su nacimiento, en versión papel, hasta su transformación en versión electrónica. Para dicho análisis nos hemos fijado en la Revista Dragón, que ha sido la revista sobre juegos de rol más importante editada hasta la fecha, especialmente en lo concerniente al primer juego de rol comercial, Dungeons \& Dragons.

Palabras clave: Revista Dragón, juegos de rol, publicaciones electrónicas, historia editorial.

AвSTRACT: This work study and analyze the history and development of a journal, from its birth, on paper, until its transformation into electronic version. For this analysis we have focused in Dragon Magazine, which was the most important magazine of role playing games (or RPGs) published until now, especially with regard to the first commercial RPG, Dungeons \& Dragons.

Keywords: Dragon Magazine, role playing games (RPGs), electronic publications, publishing history. 


\section{INTRODUCCIÓN}

En España han existido múltiples revistas dedicadas a los juegos de rol, entre las más afamadas podemos hablar de las siguientes: Troll, Sire, Líder, 2D10 (en sus dos etapas), RPG Magazine o quizá la más afamada, la revista Dragón, que también ha tenido dos etapas.

Esta última en especial, así como el resto de revistas mencionadas han «bebido» de la primera revista de envergadura que apareció en el mercado tratando el tema de los juegos de rol, la revista Dragón, en su edición original norteamericana. Hemos elegido a esta revista, no sólo por dar el salto desde el formato papel al soporte electrónico, sino también por su importancia en el desarrollo del primer juego de rol comercial, Dungeons \& Dragons. En este artículo hemos querido hacer un recorrido histórico por la «vida» de una revista dedicada a este tipo de juegos y para ello, comenzaremos centrándonos en los inicios de esta revista, para posteriormente hablar de los principales autores que se dieron a conocer gracias a la revista Dragón, continuaremos con una pequeña evolución de la misma y finalizaremos con algunas de las cubiertas de los números que, a nuestro modo de ver, han sido más importantes a lo largo de sus más de 30 años de existencia.

\section{Los COMIENZOS}

En enero de 1974 salió de imprenta el primer juego ${ }^{1}$ de Dungeons \& Dragons (a partir de ahora abreviado como $D \& D$ ), o lo que es lo mismo el primer juego comercial de rol. Fue confeccionado de forma casera y empaquetado en cajas genéricas con unas etiquetas pegadas en el exterior. Un año más tarde, en 1975, TSR (Tactical Studies Rules) Hobbies Inc. creó y comenzó a publicar en Estados Unidos un boletín llamado Revista Estratégica, que llegó a cinco números antes de verse sustituido por la revista Dragón al año siguiente, naciendo con una periodicidad mensual. En 1979, el cuartel general de la revista estaba todavía situado en una casa en el 723 de Williams Street en el Lago Ginebra, Wisconsin. Las oficinas se crearon transformando las habitaciones y los dormitorios, es decir, reconvirtiendo un hogar, en oficina de negocios.

1 No podemos hablar de libro en sentido estricto, puesto que lo primero que se editó de este juego fue una caja con 3 libros, por ello hablamos de juego y no de libro. 
Quizás uno de los ganchos de esta revista hayan sido las ilustraciones de sus «portadas», que han sabido atraer a un público joven influenciado por la literatura fantástica. Tenemos como ejemplos la portada del número $49^{2}$, realizada por el pintor Tim Hildebrandt ${ }^{3}$ (uno de los pioneros de nuestro tiempo en lo que ilustraciones sobre fantasía se refiere) o la de Boris Vallejo para el número $52^{4}$. Tampoco habría que olvidar al tristemente fallecido Keith Parkinson, que trabajó en las portadas de esta revista hasta su paso a la editorial TSR, momento en que se convirtió en una «estrella» de este género.

\section{LOS DESCUBRIMIENTOS}

El caso de Keith Parkinson no es único. También está el ejemplo de un joven, Larry Elmore, que a principios de los 80 trabajaba como ilustrador técnico para la U.S. Army en Kentucky. Tras mandar una serie de dibujos a la revista, fue contratado para encabezar un suplemento de la Dragón titulado Days of the Dragon, que fue el primero de una serie de calendarios publicados en este medio en los primeros años de la década de los 80 . En los más de 20 años transcurridos desde entonces, ha creado multitud de dibujos para la revista Dragón y para TSR, es más, actualmente uno de los mundos de D\&D no se puede concebir sin visualizar los dibujos de este autor. Estamos hablando de Dragonlance.

El ejército norteamericano forma parte también de esta historia, al haber aportado, entre otras cosas, excelentes dibujantes y buenos escritores. Esto es debido, en gran parte, a que las fuerzas armadas estadounidenses adoptaron el juego de D\&D como una estrategia más de preparación para asumir diferentes roles, activar la cooperación en grupo y potenciar la capacidad de adaptación al medio. Por ello no nos debe sorprender que el departamento

2 Aquí asistimos a una reestructuración importante de la revista al ocupar Kim Mohan el cargo de redactor jefe, en sustitución de Jake Jaquet, que a su vez pasa al cargo de editor (publisher), ocupado anteriormente por Gary Gygax. Hasta ese momento Mohan había sido asistente de redacción.

3 En este número se incluye una entrevista completa al ilustrador que puede leerse en: Knorr, Bryce: Life at least, Tim Hildebrandt's vision of life, unwinds at an unrelenting 24 frames a second. Dragon Magazine, 1981, Núm. 49, pp. 41-53.

4 Además de la cubierta, en este mismo número hay una extensa entrevista sobre este conocido dibujante, la referencia es: Knorr, Bryce: Boris. Dragon Magazine, 1981, Núm. 52, pp. 28-35. 
de las fuerzas armadas donde había mayor número de jugadores de rol fuera el de psicología. Uno de los mejores escritores y de los más prolíficos de esta revista, proviene de este campo, en concreto del servicio de psicología de la base norteamericana de Alemania. Se trata de Roger Moore, creador entre otras cosas de los panteones de enanos, elfos, medianos, gnomos y orcos para D\&D. Comenzó en la revista publicando una serie de artículos, para posteriormente convertirse en editor contribuyente y tras dejar las fuerzas armadas, llegar a ser asistente editorial.

Dicho articulista empieza a tener influencia en la revista Dragón en julio de 1983, pero es desde el número 100 cuando su nombre adquiere un peso específico, como ya mencionamos en líneas anteriores, al convertirse en asistente editorial. Con el abandono de Kim Mohan, a partir del número 115 de noviembre de 1986, pasa a ocupar el cargo de editor (que en español sería más correcto traducirlo como redactor) de las dos revistas más importantes de D\&D, es decir, de Dungeon y de Dragón.

La revista Dragón floreció con el nuevo aliento dado por Roger Moore, otorgándole un giro humorístico que hasta ahora no poseía. Gracias a él, muchos artistas de cubiertas famosos en la actualidad, comenzaron a realizar sus primeros trabajos para esta revista y para TSR en esta nueva etapa, entre ellos estarían: Carol Heyer, Charles Vess, Bob Eggleton, Jeff Easley, Gerald Brom (autor de la iconografía de Dark Sun), Fred Fields y Tobh Ruppel, por nombrar a algunos de ellos.

En este período TSR empezó a trabajar en la segunda edición de AD\&D, y la revista Dragón se convirtió en el foro apropiado para poder hablar de ello. No sorprende por tanto que David «Zeb» Cook eligiera esta tribuna para escribir una gran cantidad de artículos que hablaban de las diferentes vías que se podían seguir en el desarrollo de este juego, el D\&D, o cómo se debería elegir un camino y no otro. Incluso llegaba a exponer por qué determinada decisión editorial sobre el juego había sido tomada en detrimento de otra y cómo había sido el proceso de creación de dicha idea. Así por ejemplo se pude decir que el número 142 de la revista, que salió en el año 89, era de facto, una «preedición» de la segunda edición de $A D \& D$ (Advanced Dungeons \& Dragons).

Otro hito lo marcó el número 148, en el que se inició la publicación de cartas a todo color que podían retirarse de la publicación. Estas cartas que 
ahora nos resultan tan familiares gracias a juegos como Magic ${ }^{5}$, supusieron entonces toda una novedad. No eran cartas al uso, como las del juego mencionado anteriormente, sino que se trataban de una colección de uno de los objetos mágicos de D\&D más conocidos, el Mazo de Muchas Cosas ${ }^{6}$.

Para esta publicación también han contribuido otras leyendas de D\&D, como es el caso de Ed Greenwood, que pasó de ser un auxiliar de biblioteca en Toronto a creador del mundo con mayor éxito de todo D\&D, Reinos Olvidados. De hecho a mediados de los 80, cuando Greenwood comenzó a publicar para la revista Dragón ya resultaba evidente que todo el material que tenía escrito sería imposible de publicar por esta revista. Poseía más cantidad de mapas, más campañas y más texto de lo que nunca podría publicar este magazine. El salto a TSR parecía inevitable y sus ideas acabaron formando parte de la iconografía del rol.

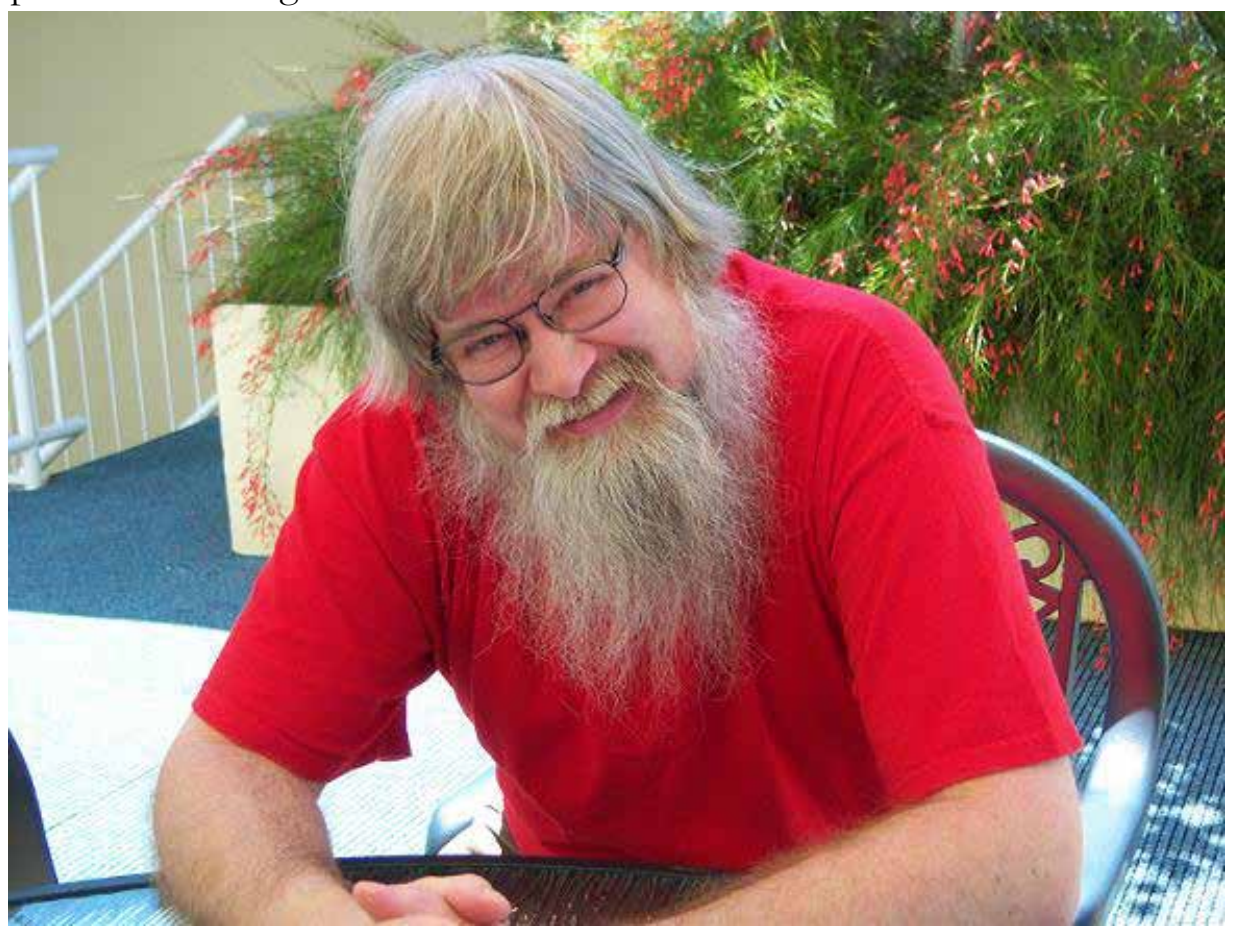

Fig.1. Fotografía de Ed Greenwood.

5 Juego de cartas coleccionables creado en 1993 por Richard Garfield y comercializado por Wizards of the Coast, actuales editores de D\&D.

6 Lo que era el «mazo» en sí, estaba inserto dentro de la publicación. Además de este, en el número 148 se incluía un artículo que explicaba cuestiones relativas a este «objeto mágico». El artículo es: Jenkins, Robin: Luck of the Draw, even prayers are useless against the deck of many things. Dragon Magazine, 1989, Núm. 148, pp. 44-52. 
A partir de la publicación del número $150 \mathrm{y}$ con la salida al mercado de la segunda edición de AD\&D se produjo una reactivación de la revista. Reflejo de todo esto eran los 12 números anuales, con un contenido aproximado de unas 120 páginas. A ello también contribuyó el lanzamiento de toda una serie de nuevos escenarios de campaña': Ravenloft, Spelljammer, Dark Sun, AlQadim, Planescape, Birthright, y la conversión de Mystara de las reglas antiguas de $\mathrm{D} \& \mathrm{D}$ a las nuevas para $\mathrm{AD} \& \mathrm{D}$. Es en estos primeros años de la década de los 90, cuando se une al proyecto Larry Smith, como director artístico de la revista, cargo que ocuparía durante 10 años.

Pero toda esta etapa se puede decir que culmina con el número 200. Tras casi 10 años a cargo de la dirección de la revista Dragón, Roger Moore, aceptó el puesto de director creativo del departamento de juegos de TSR, encargado de las reglas de la línea de AD\&D. Suponía el adiós de un hombre que había sido el corazón y el alma de la revista durante casi una década y en ese número tan sólo figura como Editorial Contribution.

\section{LA VIDA DE LA REVISTA A PARTIR DEL NÚMERO 200}

A partir de este número se realizaron numerosos cambios en donde la estabilidad del cargo no primó tanto como en los 200 números anteriores. Así tenemos por un lado a Kim Mohan quien toma el relevo dejado por Roger Moore y pasará a dirigir la revista como redactor jefe durante un año, por lo menos hasta el número 217 , y ya en mayo de 1995, cambia a TSR, a la sección de desarrollo y edición. Por la misma fecha también se crea otra vacante en la revista, la de Dale Donovan, que tras casi seis años como editor asociado pasa al departamento de desarrollo de TSR. A continuación vino un corto período en el que Wolfgang Baur se hizo con el control de la revista hasta el número 225, en el que intervino como editor Anthony J. Bryant. Bryant reorganizó la revista intentando volver a lo que habían

\footnotetext{
7 Por campaña entendemos toda una serie de aventuras (historia, expuesta delante de los jugadores para que estos interpreten a sus personajes) conectadas entre sí por un hilo argumental o dicho de otro modo, correlación de muchas aventuras con un propósito definido. El final de una campaña no se produce en una sola sesión de juego, sino en varias, dado que el objetivo que se plantea alcanzar es a largo plazo, como pueda ser rescatar varias armas mágicas para salvar un reino determinado, etc. Ver: Sevillano Pareja, Héctor: Estudio del sector editorial de los juegos de rol en España: historia, tipologia, perfil del lector, del autor, del traductor y del editor. Salamanca: Universidad de Salamanca, 2009, p. 890.
} 
sido las partes básicas de la revista: artículos de $\mathrm{PNJs}^{8}$, nuevos monstruos, conjuros, y objetos mágicos, así como algunos artículos de otros juegos que no eran D\&D. El problema vino por el intento de agradar a todo el mundo. La revista intentaba congraciarse con todos los jugadores jugasen a lo que jugasen y así no lograba contentar a nadie. Es entonces cuando Dave Gross ${ }^{9}$ llega a editor (redactor) de la revista, y con una serie de apoyos, como Brian Thomsen, Pierce Watters, Larry Smith, Michelle Vuckovich y Lizz Baldwin, intenta reconducir la publicación. El propósito no era otro que realizar una sola cosa bien todo el tiempo. La misión para la que fue contratado era la de redefinir la revista Dragón y convertirla otra vez en la referencia obligada de D\&D. Pero cuando todos estos esfuerzos estaban puestos en esta dirección, TSR expiró.

Durante más de siete meses la revista siguió publicándose, aunque sus creadores estaban esperando la llamada de algún ejecutivo que les comunicase el cierre de la revista. La revista vendía y por eso no se cerraba, aunque tampoco tanto como para no plantearse su cierre mes a mes. Los jugadores ${ }^{10}$ de D\&D asaltaban la revista con montones de preguntas sobre TSR y sobre D\&D, preguntas para las que los propios empleados de la revista Dragón no tenían respuesta.

Toda esta incertidumbre llegó a su fin con la compra de TSR por parte de Wizards of the Coast, con su director a la cabeza Peter Adkison. Se sucedieron los cambios, incluso de localización física de la sede, de Wisconsin a Seattle. Y pese al miedo inicial, la revista siguió adelante, de hecho, Adkison se reveló como un gran fan de D\&D y por ende de la revista Dragón, solicitando una columna propia. En resumidas cuentas la revista Dragón estaba salvada.

8 Pnj (Penejota o Personaje No Jugador): Los personajes no jugadores, más comúnmente conocidos como pnjs son las demás personas, monstruos o seres sobrenaturales que pueblan el mundo de juego además de los personajes de los jugadores. El que se encarga de manejarlos es el Máster. En: Sevillano Pareja, Héctor: Estudio del sector editorial de los juegos de rol en España: historia, tipología, perfil del lector, del autor, del traductor y del editor. Salamanca: Universidad de Salamanca, 2009, p. 896.

9 En el número 225 de la revista Dave Gross ya ocupaba el puesto de associate editor.

$10 \mathrm{El}$ jugador podríamos definirlo como la persona que participa en una sesión de un juego de rol llevando a un personaje e interpretándolo. Sevillano Pareja, Héctor: Estudio del sector editorial de los juegos de rol en España: historia, tipologia, perfil del lector, del autor, del traductor y del editor. Salamanca: Universidad de Salamanca, 2009, p. 893. 


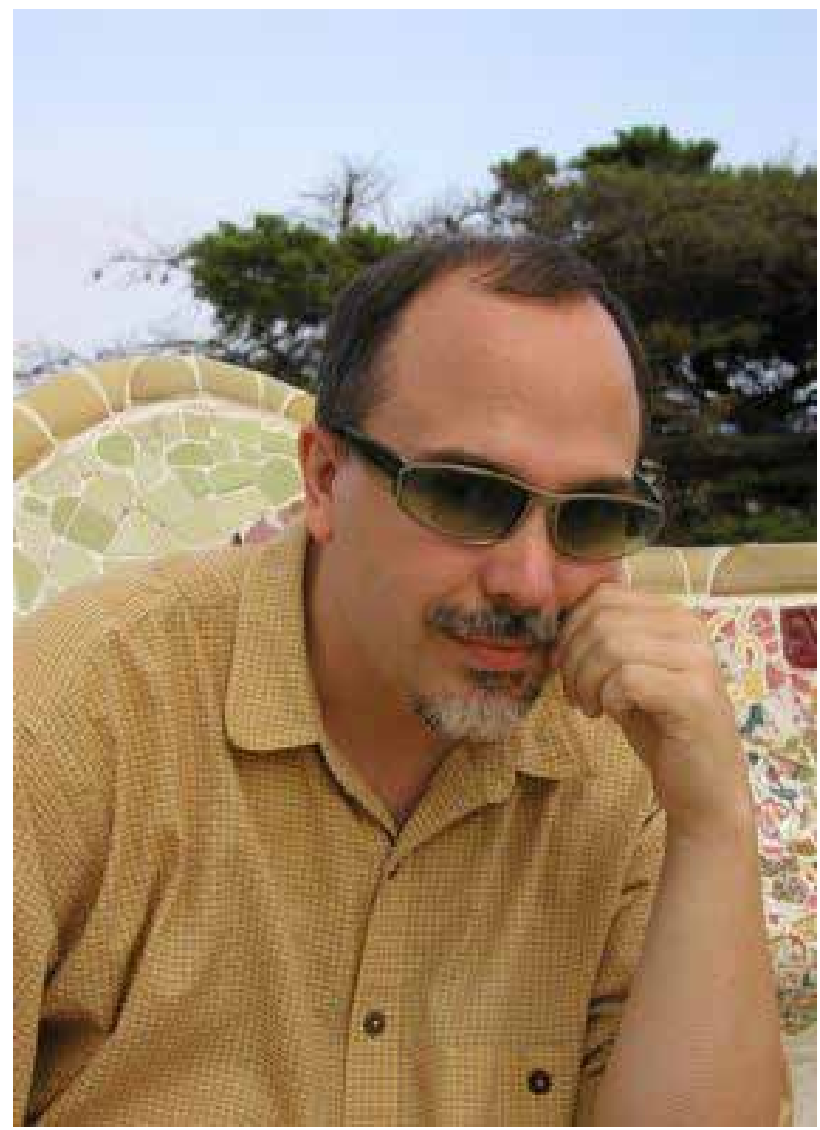

Fig.2. Fotografía de Peter Adkison.

Al contrario de lo que pudiera parecer a primera vista, las contribuciones de Adkison a la revista fueron positivas. Desde un primer momento se mostró como un gran conocedor de D\&D, y en vez de entorpecer a Gross, le dio libertad para decidir lo mejor para la revista, haciendo las sugerencias oportunas. A petición de Adkison se incrementó el presupuesto de la revista, se pasó de realizar ésta (salvo la portada y algún que otro elemento que era a color) en blanco y negro, a todo color e incluso logró que el propio Gary Gygax (coautor de la primera edición de D\&D y fundador de TSR) volviera a la palestra del juego, con una columna propia. En el fondo fue toda una vuelta a los orígenes pero con una nueva cara y mejores medios. 


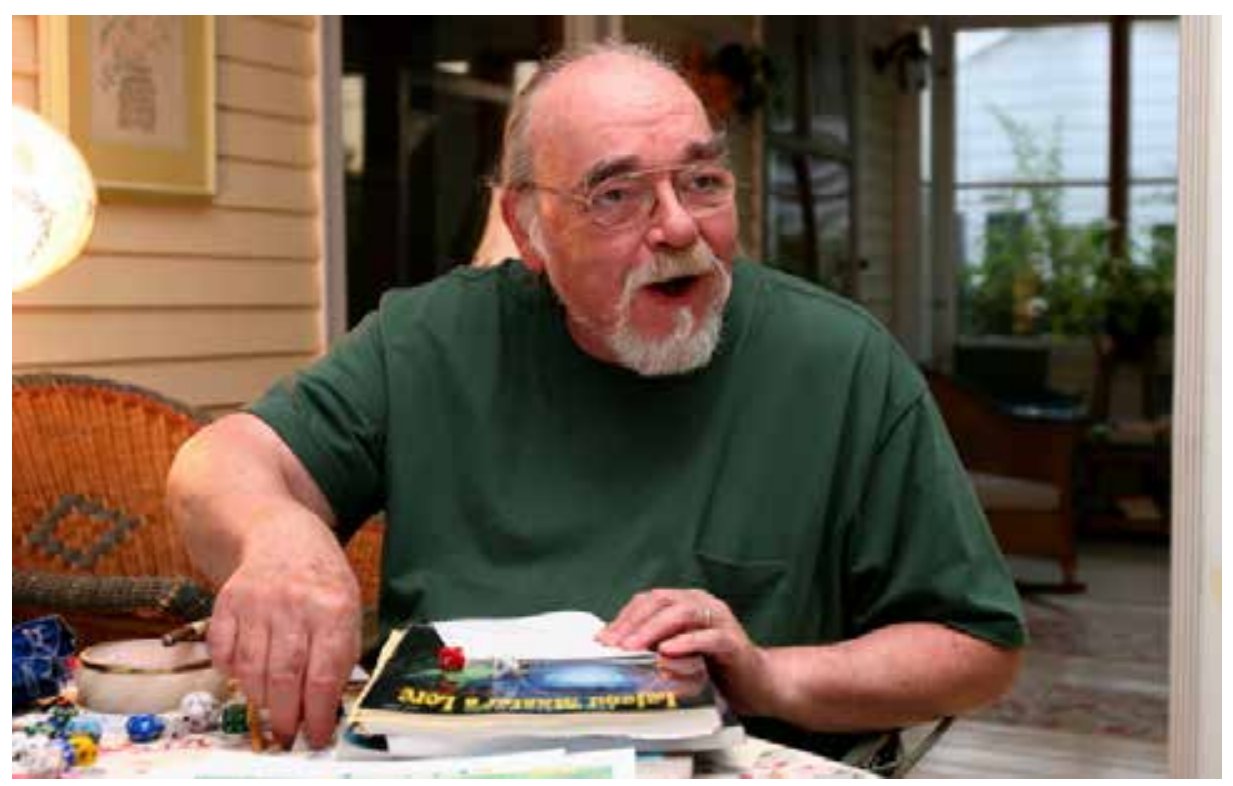

Fig.3. Fotografía de Gary Gygax.

A parte de la «inyección» monetaria y de la incorporación del presidente de la compañía al equipo, la revista Dragón, recibió una nueva serie de contribuciones gracias al cambio de compañía, como ya mencionamos, de TSR a Wizards of the Coast. La primera de ellas fue la de Christopher Perkins, prolífico colaborador de la revista Dungeon, que desde el año 1998 venía desempeñando las funciones de associate editor. A partir de finales del 2000 pasó a trabajar como contributing editor, hasta junio de 2002, momento en que comenzó una emergente carrera en Wizards, siendo en la actualidad ejecutivo del departamento de diseño de esta compañía.

Al equipo que ya venía consolidado de la etapa de TSR y tras la adicción de Perkins, habría que sumar un nuevo miembro, estamos hablando de Jesse Decker, que ocupó el puesto de asistente editorial, para a partir del número 300 sustituir a Gross como redactor jefe (Editor-in-Chief).

La segunda contribución fue la realización de una nueva edición de D\&D. Esto produjo en la revista una cierta atmósfera de vuelta a los primeros años de la publicación.

Se planteó la necesidad de rentabilizar, con un año de antelación al anuncio de su salida, la inminente $3^{a}$ edición. Había varias vías posibles sobre cómo 
podía el magazine rentabilizar esta información. Una nueva edición de D\&D en el fondo significaba volver a los orígenes de la revista. Todos aquellos artículos de los primeros años de existencia de la revista volvían a tener vigencia. En el fondo se trataba de analizar las demandas de los lectores de los primeros años de la revista y anticiparse a las que surgieran con la nueva tercera edición. Los jugadores del nuevo $\mathrm{D} \& \mathrm{D}$ querrían y necesitarían una guía sobre cómo usar las nuevas reglas, cómo crear sus propios monstruos, conjuros, objetos mágicos, y todo un nuevo conjunto de normas, como las habilidades o las clases de prestigio.

Todos estos elementos también sirvieron para que «viejas secciones», como las tiras cómicas, se consolidaran como un referente en la revista. Estaban las tiras de Phil Foglio, denominadas «Phil and Dixie»; Aarón Williams con «Nodwick» y por último las premiadas «Dork Tower» de John Kovalic.

Para comprobar si los lectores reaccionaban positivamente a estos esfuerzos, la revista sacó un artículo denominado Cuenta atrás para la $3^{a}$ edición, estamos hablando de febrero de 2000 y del número $268^{11}$ del veterano magazine. El resultado fue el aumento de cartas y de e-mails recibidos, así como un mayor número de lectores. Por otro lado Pierce Watters, que había sido redactor jefe se convirtió en director de ventas (circulation director) y procuró desde este nuevo puesto aumentar el número de stands en los que estuviera la publicación. A su vez el director de producción (production manager) John Dunn, intentó mejorar el precio final de venta y los circuitos de producción.

Por estas fechas, el hasta aquel momento director artístico (art director) Larry Smith, decidió retirarse y le cedió el puesto a Peter Whitley quién supo conciliar el nuevo estilo de la $3^{\mathrm{a}}$ edición de $\mathrm{D} \& \mathrm{D}$ con un aspecto diferente de la revista.

Quizás la contribución más crucial vino de fuera del plantel de la propia revista. Durante el año que hubo entre las pruebas de juego de la nueva versión y la publicación de la $3^{a}$ edición de D\&D y la Dragón número 274 , cientos de jugadores escribieron expresando su opinión sobre múltiples temas, recuperando el viejo contacto entre jugadores, juego y revista tan propio de los primeros años de la publicación. Muchas de estas cartas con sugerencias tuvieron un gran efecto en la versión final de la nueva edición

11 Noritake, Wendy (Ed.): Countdown to 3rd edition! Dragon Magažine, 2000, Núm. 268, pp. 24-25. 
del juego, así como en la línea editorial y los contenidos de esta renovada Dragón Magazine. Como todo, algunos antiguos lectores lamentaron los nuevos contenidos así como la cobertura de otros juegos, pero muchos otros se suscribieron a la revista. Por las cartas recibidas, la dirección de la publicación llegó a la conclusión de que una buena parte de los nuevos suscriptores o lectores de la revista eran desilusionados veteranos de juegos de rol que gracias a esta nueva versión volvía a retornar a su afición.

Es en este momento, quizás el mejor de la revista, cuando los hasta entonces principales «adalides» de la misma decidieron que era el tiempo indicado para que las nuevas generaciones de jugadores tomaran el relevo y llevaran a la publicación a nuevas metas. Dave Gross dimitió de su cargo como editor jefe en septiembre de $2001^{12}$ pasando a dedicarse a la escritura de libros de literatura fantásticas (aparte de alguna contribución suelta en la revista) y Christopher Perkins, en el año 2001, se dedicó únicamente a realizar artículos. En junio de $2002^{13}$ dejaba definitivamente la revista para integrarse en Wizards.

Desde entonces y hasta el final de la revista los cambios se fueron sucediendo, el último número editado por Paizo y autorizado por la licencia de Wizards salió a la venta en septiembre de $2007^{14}$ con el dígito 359. La revista Dragón dejó de editarse al romper Wizards of the Coast el acuerdo que tenía con Paizo para la publicación de la versión en inglés (así como los de la revista Dungeon). La razón alegada por Wizards era que los contenidos de la revista Dragón se buscaban más en la red que en la propia revista, así que el contenido de dicha revista pasaría a publicarlo directamente Wizards

12 La carta de renuncia se puede leer en la página 6 de esta revista. La referencia bibliográfica completa es: Gross, Dave: Dear Dragon. Dragon Magazine, 2001, Núm. 287, p. 6.

13 A partir de agosto de 2002 la titularidad de la revista Dragón pasó de Wizards of the Coast a Paizo Publishing, en una maniobra económicamente poco clara, puesto que la tirada de la revista era entonces de 70.000 ejemplares, es decir, era económicamente rentable. Pese a este cambio la revista siguió conservando el título de Revista Oficial de Dungeons \& Dragons y por tanto se consideraba que cualquier material publicado en esta revista era material oficial de dicho juego. De hecho las oficinas de esta revista estaban integradas dentro del edificio de Wizards y muchos de los autores de los artículos de dicha revista trabajaban para esta última compañía. De todas maneras si se quiere saber algo más sobre esta revista es conveniente consultar: Decker, Jesse: Readers talk back, Scale mail. Dragon Magazine, 2002, Num. 300, pp. 8-10.

14 En realidad apareció en los comercios el 29 de agosto, aunque correspondía al número del mes de septiembre. 
en la red, saltándose de paso al intermediario que era Paizo, al que le tenía vendidos los derechos de licencia de las revistas Dragón y Dungeon ${ }^{15}$.

Durante el último periodo de la revista, el puesto de editor jefe tuvo varias caras desde Dave Gross, a Jesse Decker hasta Erik Mona, que fue el último. De la «vieja guardia» solamente se mantuvo Pierce Watters, que conservó su cargo de director de ventas.

\section{Vida MÁS ALLÁ DEL PAPEL}

En la actualidad la revista Dragón, como tal en formato papel, ha desaparecido y ha sido sustituida por una versión on-line editada por Wizards of the Coast (al igual que ha ocurrido con la revista Dungeon), y cuya consulta (y/o descarga) se puede realiza a partir de la web de esta editorial en el apartado D\&D Insider ${ }^{16}$. Además cuenta con una recopilación anual de los mejores artículos aparecidos en este nuevo soporte, pero en formato libro y ya no revista, denominada Dragon Magazine Annual, con un coste de 29,95\$ y del que ya han aparecido dos números en el mercado, publicándose el primero de ellos el 18 de septiembre de 2009, es decir prácticamente un año después del cierre de la revista en papel. ¿Qué ofrece el anuario? Pues lo que ha ofrecido siempre la revista Dragón, nuevas opciones para los personajes, información para el Máster sobre los mundos ${ }^{17}$ oficiales de juego, siendo todo este material totalmente compatible con las reglas de juego de D\&D

15 Un extracto de la noticia se puede leer en: Bowesley: Fin de las revistas Dungeon y Dragón. Templo de Hecate. 14 de abril de 2007. [En línea].

$<$ http://www.templodehecate.com/noticia/1311/fin de las revistas dungeon y dragon.html $>$ [Consulta: 7 de marzo de 2011]. También es posible encontrar en la red el escrito conjunto de Paizo y Wizards of the Coast, en donde comunica el fin de las revistas Dragón y Dungeon en papel, la dirección es: Paizo Publishing. News. Dragon. Press Releases: Paizo Publishing to Cease Publication of Dragon and Dungeon. 19 de abril de 2007. [En línea].

$<$ http://paizo.com/paizo/news/dragon/pressReleases/v5748eaic9kh0 $>$ [Consulta: 7 de marzo de 2011].

16 La suscripción a D\&D Insider da acceso a varias aplicaciones de juego, como un editor de personajes jugadores, una base de datos de monstruos de juego, una herramienta de creación de aventuras, y acceso a las revistas on-line Dragon Magazine y Dungeon Magazine. Todo ellos desde 9,95\$ al mes o 71,40\$ si se paga una anualidad (también existe la opción de pago por trimestre a 23,85\$). Para más datos consultar: Wizards of the Coast. D\&D. Home: Subscribe to D\&D Insider. 7 de marzo de 2011. [En línea].

$<$ http://www.wizards.com/DnD/Subscription.aspx > [Consulta: 7 de marzo de 2011].

17 Es decir, la ambientación de juego en donde se desarrolla la partida. Sevillano Pareja, Héctor: Estudio del sector editorial de los juegos de rol en España: historia, tipologia, perfil del lector, del autor, del traductory del editor. Salamanca: Universidad de Salamanca, 2009, p. 895. 
y $100 \%$ oficial. La novedad sin embargo estriba en que ahora aparecen las notas de diseño, de desarrollo y del redactor, para que el lector pueda ver cuál ha sido el origen de cada artículo y su evolución.

Como curiosidad habría que reseñar que cada número on-line, con periodicidad mensual, continua con la numeración original que poseía la revista Dragón, yendo ya por el número 397 a día 7 del mes del marzo de 2011.

\section{Principales cubiertas}

En este punto hemos incluido aquellas cubiertas que pueden ser más representativas. Por motivos obvios hemos puesto la primera que salió a la venta (ver fig. 4), allá por el año 1976 y el cambio con respecto al último número publicado en papel, más de treinta años después, es evidente, no solo en estructuración sino también en la calidad del dibujo. Estamos hablando del número 359, del año 2007 (ver fig. 9).

Entre medias de estas dos imágenes, hemos puesto las de todos los centenarios, pues al ser números especiales las publicaciones suelen esmerarse más e intentan hacerlas más espectaculares. Ejemplo de esto son los números 100, 200 (fig. 6) y 300. El primero de ellos (ver fig. 5) es una suerte de juego de volúmenes, pues el dragón del centro de la imagen es en realidad una figura creada en papel en tres dimensiones por Dennis Kauth. En cuanto al último, se trata de un gran dragón áureo, trabajo de Lisa Chido, que representa magníficamente el espíritu de una revista como es Dragón. Hemos puesto la cubierta (ver fig. 7) y el dibujo original (ver fig. 8) para que pueda apreciarse el trabajo de encajar el texto sin estropear la pintura, al llevar a cabo la maquetación de la «portada». 


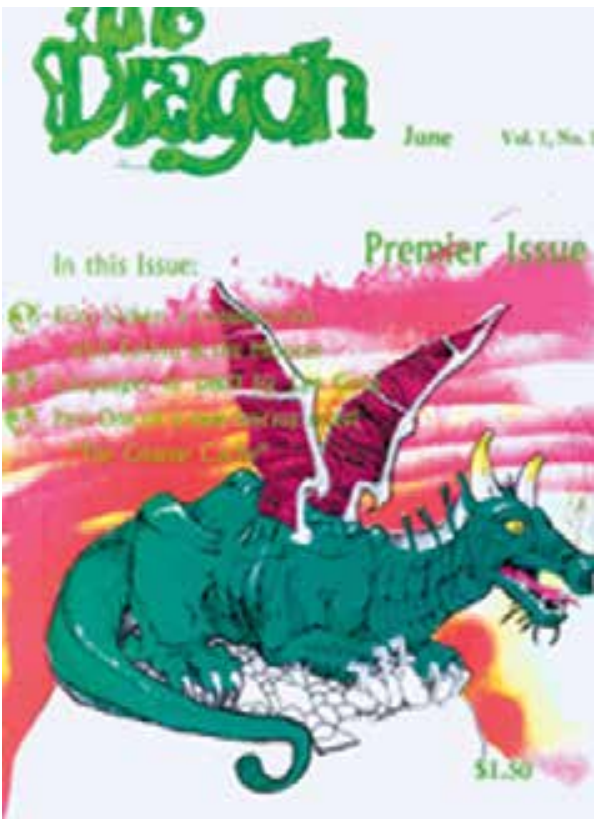

Fig. 4. TSR, No 1 , junio de $1976^{18}$.

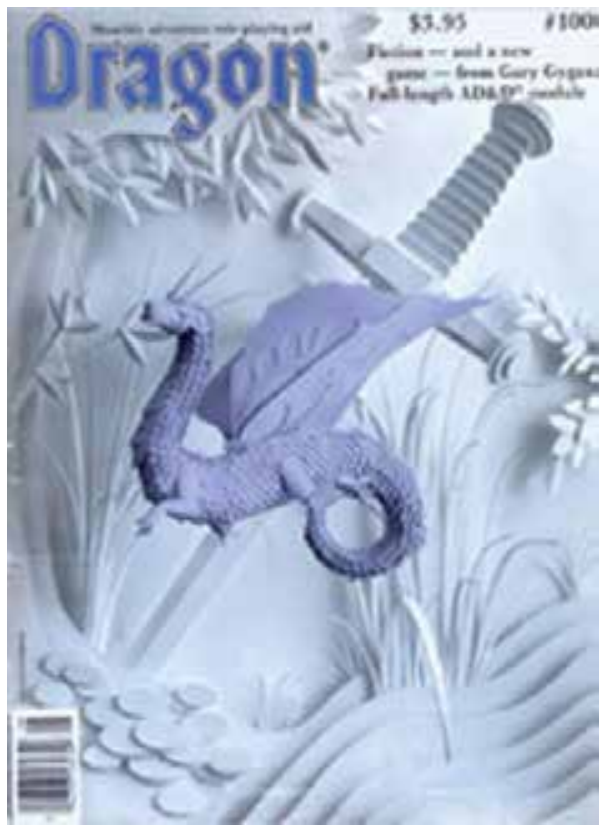

Fig. 5. TSR, No 100, agosto de 1985.

18 Los pie de fotografía de las cubiertas que aquí mostramos los hemos organizado mencionando en primer lugar la editorial responsable de su publicación, a continuación el número de la revista (en el caso de que posea uno), y por último la fecha de publicación. 


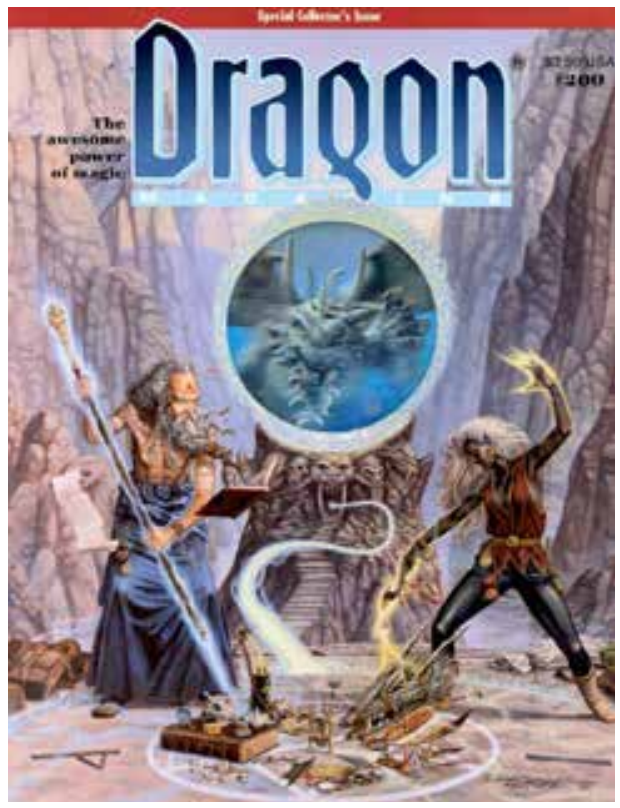

Fig. 6. TSR, No 200, diciembre de 1993.

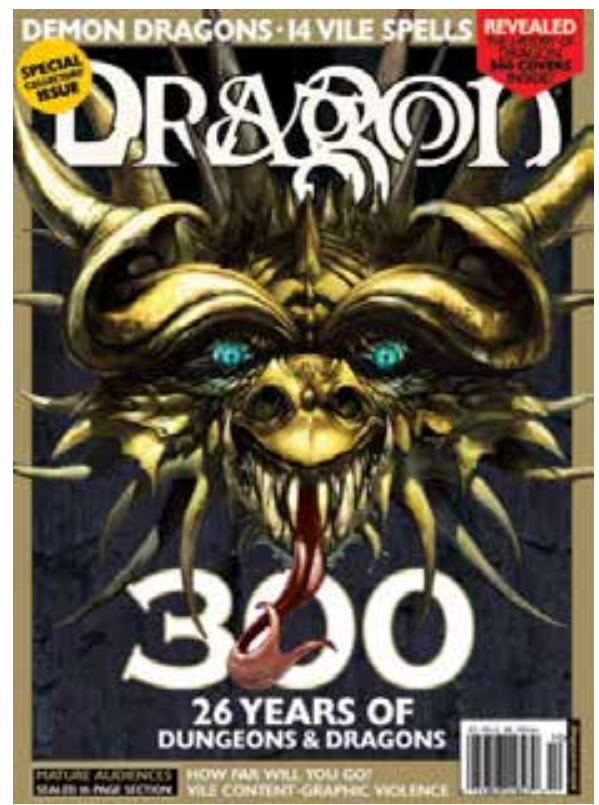

Fig. 7. Paizo Publishing, $\mathrm{N}^{\circ} 300$,

octubre de 2002.

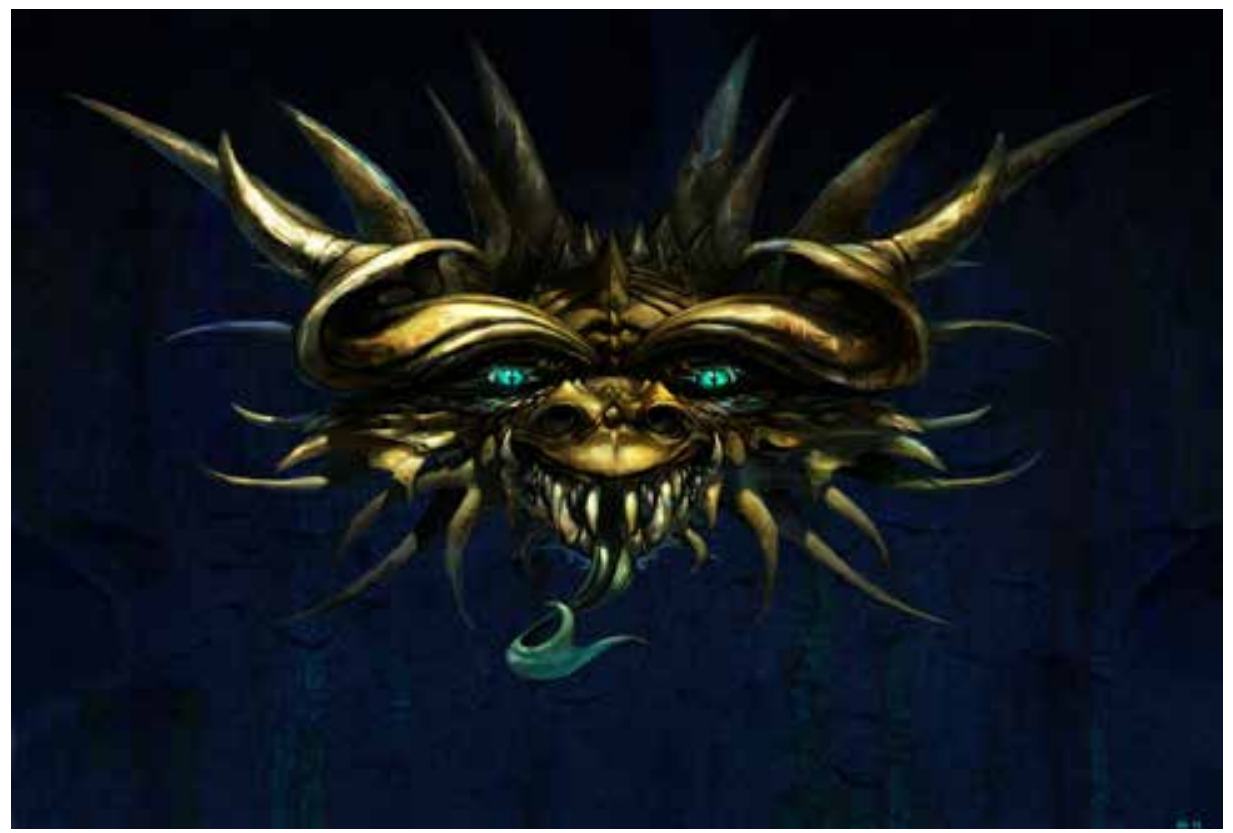

Fig. 8. Ejemplo de dibujo para una portada: Dragón dorado, por Lisa Chido. 


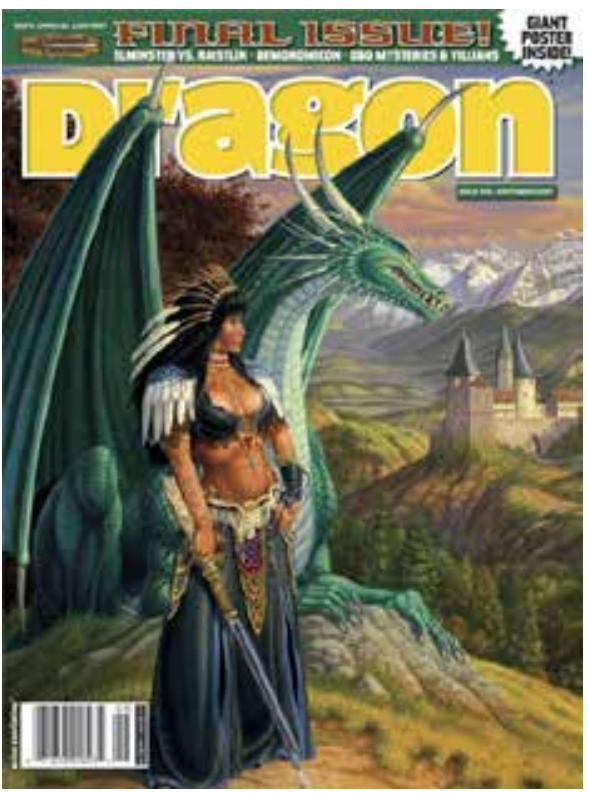

Fig. 9. Último número antes del cierre de la revista: Paizo Publishing, No 359, septiembre de 2007.

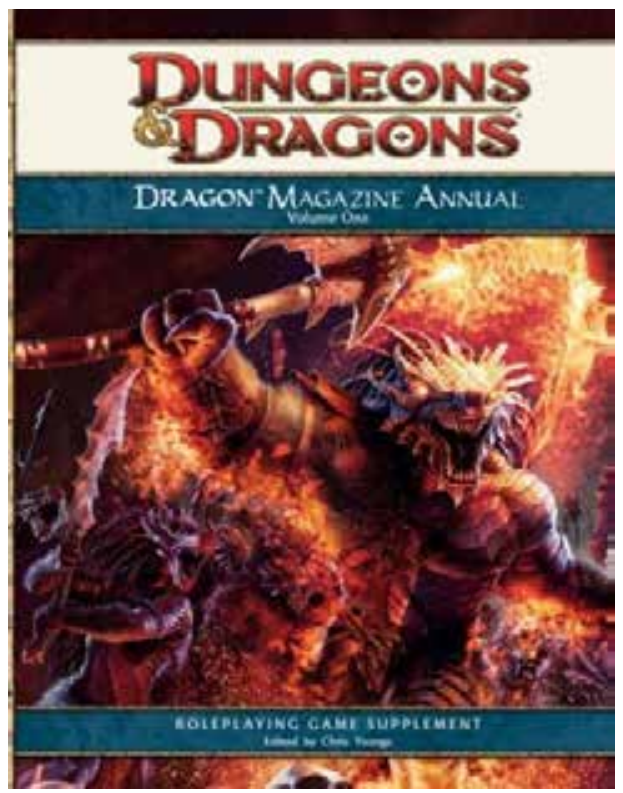

Fig. 10. La «revista» Dragón en formato libro: Wizards of the Coast, Dragon Magazine Annual 2009.

Por último hemos incluido el primer número del Dragon Magazine Annual (fig. 10), editado por Chris Youngs, acompañado de un número de la edición on-line (en este caso el número 368, fig. 11) dado que ambos comparten el mismo dibujo, el creado por Steve Argyle en 2008 por encargo de Wizards of the Coast y denominado «Dragonborn Barbarians» (ver fig. 12). 


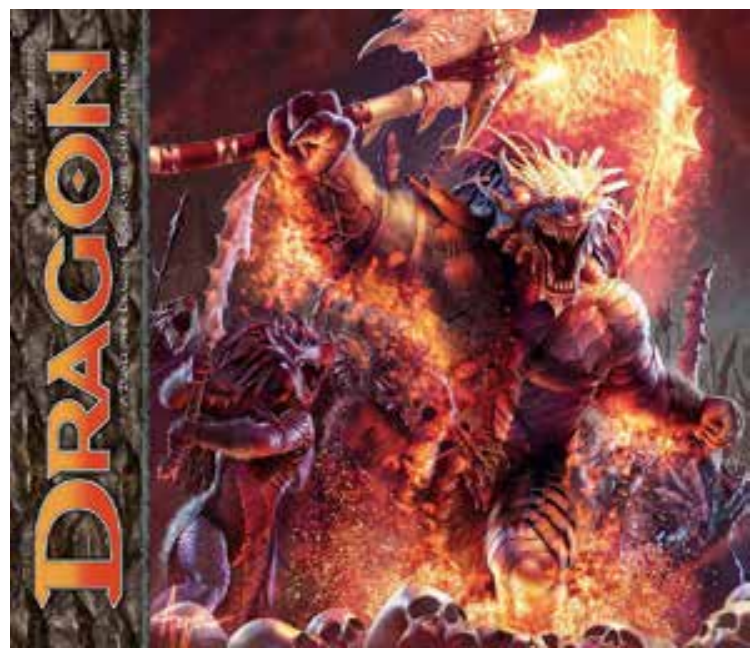

Fig. 11. La «revista» Dragón en su versión on-line: Wizards of the Coast, $\mathrm{N}^{\mathrm{o}}$ 368, octubre de 2008.

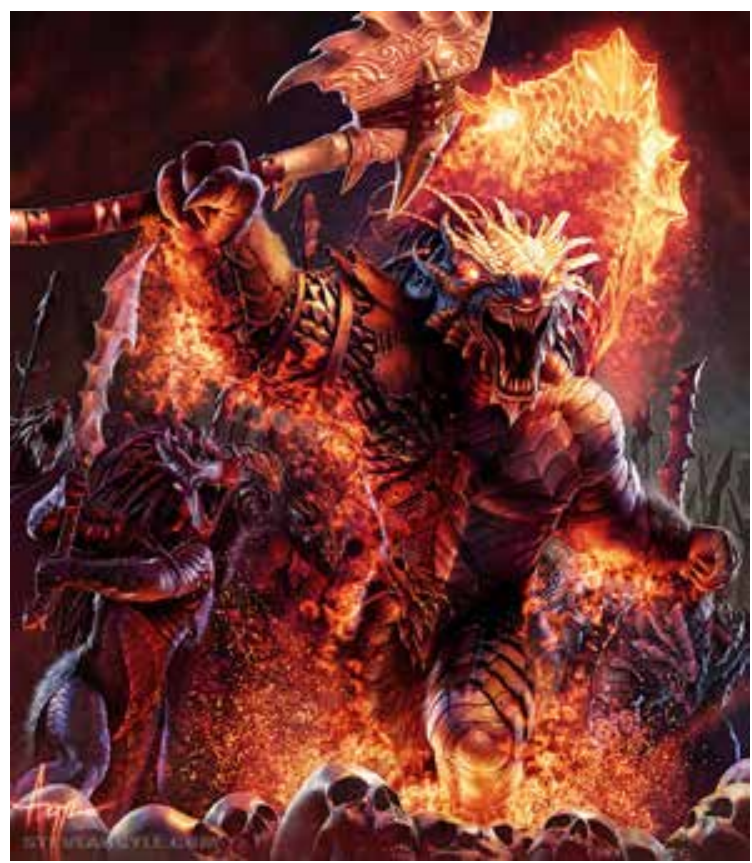

Fig. 12. Ejemplo de dibujo para una portada: Dragonborn Barbarians, 2008 por Steve Argyle.

Dibujo para la cubierta del Dragon Magazine Annual, Volumen 1 de la $4^{\mathrm{a}} \mathrm{ed}$. D\&D. 


\section{Bibliografía}

Bowesley: Fin de las revistas Dungeon y Dragón. Templo de Hecate. 14 de abril de 2007. [En línea].

<http://www.templodehecate.com/noticia/1311/fin de las revistas dungeon y dragon.html $>$ [Consulta: 7 de marzo de 2011].

Butterfield, John; Honigmann, David; Parker, Philip: What is dungeons and dragons.

Harmondsworth: Puffin Books, 1984.

Cook, Mike (Ed.): Dragon, 1985, Núm. 100.

Cook, Mike (Ed.): Dragon Magazine, 1986, Núm. 115.

Cook, Mike (Ed.): Dragon Magazine, 1989, Núm. 142.

Cook, Mike (Ed.): Dragon Magazine, 1989, Núm. 150.

Cottrill, Torah; Horner, Miranda; Youngs, Chris: Dragon Magazine Annual. Renton (Wa): Wizards of the Coast, 2010.

Eckelberry, David; Mohan, Kim; Winter, Steve: The story of TSR, 1975-1999. Renton

(Wa): Wizards of the Coast, 1999.

Gross, Dave: Dear Dragon. Dragon Magazine, 2001, Núm. 287, p. 6.

Jenkins, Robin: Luck of the Draw, even prayers are useless against the deck of many things. Dragon Magazine, 1989, Núm. 148, pp. 44-52.

Kask, Timothy J. (Ed.): The Dragon, 1976, Núm. 1.

Knorr, Bryce: Boris. Dragon Magazine, 1981, Núm. 52, pp. 28-35.

Knorr, Bryce: Life at least, Tim Hildebrandt's vision of life, unwinds at an unrelenting 24 frames a second. Dragon Magazine, 1981, Núm. 49, pp. 41-53.

Mohan, Kim; Donovan, Dale: Dragon Magazine, 300 issues of Roleplaying history. Dragon Magaqine, 2002, Núm. 300, pp. 18-26. 
Mohan, Kim (Redactor de desarrollo): Dragon, 2007, Núm. 360.

Mona, Erik (Ed.): Dragon Magažine, 2007, Núm. 359.

Noritake, Wendy (Ed.): Countdown to $3^{\text {rd }}$ edition! Dragon Magazine, 2000, Núm. 268, pp. 24-25.

Noritake, Wendy (Ed.): Dragon Magazine, 2000, Núm. 274.

Paizo Publishing. News. Dragon. Press Releases: Paizo Publishing to Cease Publication of Dragon and Dungeon. 19 de abril de 2007. [En línea].

$<\underline{\text { http://paizo.com/paizo/news/dragon/pressReleases/v5748eaic9kh0 }>}$ [Consulta: 7 de marzo de 2011].

Sevillano Pareja, Héctor: Estudio del sector editorial de los juegos de rol en España: bistoria, tipologia, perfil del lector, del autor, del traductor y del editor. Salamanca: Universidad de Salamanca, 2009.

Thomsen, Brian (Ed. Asoc.): Dragon Magazine, 1993, Núm. 200.

Thomsen, Brian (Ed. Asoc.): Dragon Magazine, 1995, Núm. 217.

Thomsen, Brian (Ed. Asoc.): Dragon Magazine, 1996, Núm. 225.

Winter, Steve (Redactor Jefe): Dragon, 2010, Núm. 394.

Youngs, Chris (Ed.): Anuario de la revista Dragón. Barcelona: Devir Iberia, 2011.

Youngs, Chris (Ed.): Dragon Magazine Annual 2009. Renton (Wa): Wizards of the Coast, 2009.

Youngs, Chris (Redactor Jefe): Dragon, 2008, Núm. 368. 